\title{
Evaluación clínica y ultrasonográfica de la glándula tiroides en pacientes con artritis reumatoide
}

\author{
Mauricio Figueroa-Sánchez, ${ }^{1}$ Lourdes Núñez-Atahualpa, ${ }^{1}$ Mariana Hernández-Zúñiga, ${ }^{1}$ \\ Beatriz Teresita Martín-Marquez, ${ }^{2}$ Erika Aurora Martínez-García, ${ }^{2}$ Eduardo Gómez-Bañuelos, ${ }^{2}$ \\ Rosa Elena Navarro-Hernández² y Mónica Vázquez-Del Mercado² \\ ${ }^{1}$ Hospital Civil "Fray Antonio Alcalde", Servicio de Radiología e Imagen; '2Universidad de Guadalajara, Centro Universitario de Ciencias de la Salud, \\ Instituto de Investigación en Reumatología y del Sistema Músculo Esquelético. Jalisco, México
}

\begin{abstract}
Resumen
Introducción: Los pacientes con artritis reumatoide pueden desarrollar enfermedad tiroidea autoinmune (ETA), cuyo diagnóstico clínico puede ser difícil debido a que ambas comparten síntomas como artralgias, mialgias, rigidez matutina o fatiga. Objetivo: Determinar la prevalencia de ETA en pacientes con artritis reumatoide. Método: Estudio transversal que incluyó 78 pacientes con artritis reumatoide y 81 controles clínicamente sanos pareados por edad y sexo. A ambos grupos se realizó cuantificación de anticuerpos antitiroideos, pruebas de función tiroidea, ultrasonido y biopsia de glándula tiroides cuando la puntuación de Thyroid Imaging Reporting and Data System (TIRADS) fue $\geq 4$. Resultados: $24.4 \%$ de los pacientes con artritis reumatoide presentó hipotiroidismo $(p=0.003)$ y altos títulos de anticuerpos antitiroideos versus controles clínicamente sanos; 53 \% de los ultrasonidos tiroideos resultó normal en pacientes hipotiroideos; en pacientes con artritis reumatoide positivos para anticuerpos antitiroideos se encontró perfusión incrementada en $40 \%$. Los casos clasificados como TIRADS 4 fueron enviados a aspiración, con resultado histopatológico benigno. Conclusiones: Se demostró el valor clínico agregado de la evaluación tiroidea en pacientes con artritis reumatoide, conforme a la prevalencia de hipotiroidismo subclínico, positividad de anticuerpos antitiroideos y anomalías en el ultrasonido independientes de la función tiroidea normal o alterada.
\end{abstract}

PALABRAS CLAVE: Artritis reumatoide. TIRADS. Hipotiroidismo.

\begin{abstract}
Introduction: Patients with rheumatoid arthritis can develop autoimmune thyroid disease (ATD), the clinical diagnosis of which can be difficult because both entities share symptoms such as arthralgia, myalgia, morning stiffness or fatigue. Objective: To determine the prevalence of ATD in patients with rheumatoid arthritis. Method: Cross-sectional study that included 78 patients with rheumatoid arthritis and 81 clinically healthy controls matched by age and gender. Both groups underwent anti-thyroid antibodies quantification, thyroid function tests, thyroid ultrasound and thyroid gland biopsy when the Thyroid Imaging Reporting and Data System (TIRADS) score was $\geq 4$. Results: Hypothyroidism was found in $24.4 \%$ of patients with rheumatoid arthritis ( $p=0.003$ ), as well as high titers of anti-thyroid antibodies versus clinically healthy controls; $53 \%$ of thyroid ultrasounds were normal in hypothyroid patients, and increased perfusion was found in $40 \%$ of rheumatoid arthritis patients who tested positive for anti-thyroid antibodies. Cases classified as TIRADS 4 underwent aspiration with benign histopathological results. Conclusions: Thyroid assessment added clinical value was demonstrated in patients with rheumatoid arthritis, according to the prevalence of subclinical hypothyroidism, anti-thyroid antibodies positivity and ultrasound abnormalities, regardless of normal or altered thyroid function.
\end{abstract}

KEY WORDS: Rheumatoid arthritis. TIRADS. Hypothyroidism.

Correspondencia:

Mónica Vázquez-Del Mercado

E-mail: dravme@ hotmail.com
Fecha de recepción: 14-07-2017

Fecha de aceptación: 21-05-2018

DOI://dx.doi.org/10.24875/GMM.18003556
Gac Med Mex. 2018;154:432-437

Disponible en PubMed

www.gacetamedicademexico.com 


\section{Introducción}

La artritis reumatoide (AR) es una enfermedad autoinmune crónica sistémica caracterizada por daño a la sinovia articular mediada por la expresión de múltiples citocinas, tales como las interleucinas (IL) 1 y 6 , el factor de necrosis tumoral (TNF)- $\alpha$, las metaloproteasas y otras moléculas. ${ }^{1,2}$

Los pacientes con AR pueden desarrollar enfermedad tiroidea autoinmune (ETA), ,3 la cual abarca la enfermedad de Graves (EG), tiroiditis autoinmune crónica, hipotiroidismo subclínico y tiroiditis de Hashimoto $(\mathrm{TH}) .^{5}$ En los pacientes con AR puede ser difícil establecer la sospecha clínica de ETA debido a que comparten síntomas como artralgias, mialgias, rigidez matutina 0 fatiga. ${ }^{6}$

La ETA puede desarrollar autoanticuerpos contra peroxidasa tiroidea (anti-TPO) (13 a $40 \%$ ) y tiroglobulina (anti-Tg) (23\%), ${ }^{4,7-11}$ De 4 a $37 \%$ de los pacientes con ETA muestran anti-TPO y 6 a $30 \%$ anti-Tg ${ }^{4,12}$ Se ha registrado que la prevalencia de ETA y disfunción tiroidea en pacientes con AR es de 6 a $37 \%$.5,6,8

Se informa poco sobre la utilidad del ultrasonido (US) de la glándula tiroidea como herramienta diagnóstica en la evaluación clínica de los pacientes con AR, con o sin disfunción tiroidea conocida. Aunado a esto, es inusual la realización de biopsias tiroideas por aspiración.

El propósito del presente estudio fue determinar la prevalencia de ETA mediante la detección de anticuerpos antitiroideos, pruebas funcionales, US y biopsia por aspiración de glándula tiroides de acuerdo con el Thyroid Imaging Reporting and Data System (TIRADS) en pacientes con AR y en controles clínicamente sanos (CCS) pareados por edad y sexo (controles).

\section{Método}

El protocolo fue aprobado por el comité de ética del Hospital Civil "Dr. Juan I. Menchaca", Universidad de Guadalajara, Jalisco, México, con registro 1068/10. Se obtuvo el consentimiento informado por escrito de todos los pacientes.

Se reclutaron 78 pacientes con AR que acudieron al Servicio de Reumatología del hospital entre 2010 y 2013. Debían ser mayores de 18 años y cumplir con los criterios del American College of Rheumatology (ACR, 1987) o ACR/European League Against Rheumatism (ACR/EULAR, 2010). ${ }^{13,14}$ Se excluyeron los pacientes que se encontraban con tratamientos de altas dosis de esteroides (> $15 \mathrm{mg} /$ día de prednisona o su equivalente). Como CCS se tomaron en cuenta 81 donadores de sangre, pareados por edad y sexo. La historia de enfermedad cardiovascular, hipertensión, diabetes mellitus, daño renal o hepático, cáncer, dislipidemia conocida y menopausia prematura se consideraron criterios de exclusión en ambos grupos.

Se aplicó un cuestionario a cada individuo para obtener información clínica y demográfica. La actividad de la enfermedad fue determinada por los niveles de proteína $C$ reactiva, de acuerdo con el Disease Activity Score 28 (DAS28). ${ }^{15}$

En suero se midió la triyodotironina libre (T3L) y la tiroxina libre (T4L), mediante inmunoquimioluminiscencia. De acuerdo con los niveles séricos de la hormona estimulante de la tiroides (TSH), la disfunción tiroidea se clasificó de la siguiente forma: ${ }^{16}$

- Eutiroidismo, TSH de 0.4 a $4.0 \mathrm{mU} / \mathrm{L}$.

- Hipotiroidismo abierto, TSH > $4.0 \mathrm{mU} / \mathrm{L}$ y $\mathrm{T} 4 \mathrm{~L}$ bajo.

- Hipotiroidismo subclínico, TSH > $4.0 \mathrm{mU} / \mathrm{L}$ y T4L normal,

- Hipertiroidismo, TSH $<0.4 \mathrm{mU} / \mathrm{L}$.

Se midió la velocidad de sedimentación globular $(\mathrm{mm} / \mathrm{h})$ por el método de Wintrobe. Los niveles de proteína $C$ reactiva fueron cuantificados por nefelometría. El factor reumatoide (FR) se determinó por turbidimetría. Los anticuerpos antipéptido cíclico citrulinado (anti-PCC, Axis-Shield Diagnostic, Dundee, Escocia), anticuerpos anti-TPO y anticuerpos anti-Tg (Orgentec Diagnostika, Mainz, Alemania) se midieron mediante ensayo de inmunoabsorción ligada a enzimas (ELISA).

La evaluación por ultrasonido se llevó a cabo mediante un US de alta resolución en modo $B$ con un transductor de $9 \mathrm{MHz}$. Tres radiólogos realizaron la evaluación y clasificación de las imágenes de acuerdo con TIRADS: ${ }^{17,18}$

- TIRADS 1, glándula tiroidea normal.

- TIRADS 2, lesiones benignas.

- TIRADS 3, probabilidad de lesiones benignas, usualmente en $\mathrm{TH}$.

- TIRADS 4-6, lesiones con incremento del riesgo de cáncer.

A los pacientes clasificados como TIRADS $\geq 4$ se les realizó biopsia por aspiración con aguja fina de 22 $G$ y una jeringa de $10 \mathrm{~mL}$ guiada por US. El aspirado se fijó con alcohol a $96 \%$ y se tiñó con hematoxilina-eosina. Los exámenes citológicos los llevó a cabo un patólogo experto de acuerdo con el sistema de Bethesda. ${ }^{19,20}$ 


\section{Análisis estadístico}

Los datos se analizaron con el programa estadístico SPSS versión 24.0 (SPSS Inc., Chicago, IL, EE. UU.) y GraphPad Prism versión 6.00 (GraphPad Software, La Jolla California, EE. UU.). La distribución normal de las variables se llevó a cabo con las pruebas Skewness y Kurtosis. Las variables continuas con distribución normal se calcularon con t de Student para muestras no pareadas; en distribución no normal, las variables se compararon con $U$ de Mann-Whitney. La asociación entre variables categóricas se analizó mediante chi cuadrado o por prueba exacta de Fisher. Se consideró como un valor significativo una $p \leq 0.05$.

\section{Resultados}

En la Tabla 1 se muestran las características clinicas y demográficas de los casos incluidos. Se incluyeron 78 pacientes con AR, $83.3 \%$ del sexo femenino. El promedio de edad y duración de la enfermedad fue de $42.75 \pm 12.15$ años y $4.74 \pm 6.04$ años, respectivamente.

El hipotiroidismo subclínico fue la disfunción tiroidea más frecuente en pacientes con AR. El hipotiroidismo se encontró en $24.4 \%$ de los pacientes con AR versus $1.2 \%$ de los CCS ( $p=0.003)$. En el grupo de AR con disfunción tiroidea, de acuerdo con los niveles de TSH y T4L, $89.5 \%(n=17 / 19)$ presentaba hipotiroidismo subclínico y $10.5 \%(n=2 / 19)$ hipotiroidismo franco (datos no mostrados).

Los pacientes presentaron niveles elevados de anticuerpos anti-TPO (118.26 \pm 538.60 versus $19.18 \pm 55.28, p=0.02)$ y anti-Tg $(96.94 \pm 321.27$ versus $54.99 \pm 46.43, p=0.04$ ) comparados con CCS. Se observaron nódulos tiroideos en $23 \%$ de los pacientes versus $28.4 \%$ ( $p=0.13$ ) en CCS. La categoría TIRADS 1 fue más frecuente, seguida por TIRADS 2, $4 a, 3$ y $4 b$.

En la Tabla 2 se describen los pacientes que presentaron anticuerpos anti-TPO, los cuales positivos para anti-PCC en $83.3 \%, p=0.04$, y FR en $75 \%$, $p=0.03$. Se observó perfusión incrementada en $40 \%$ de los pacientes positivos para anticuerpos anti-TPO y anti-Tg. Aproximadamente $30 \%$ de los pacientes que clasificaron en TIRADS 3 mostraron anticuerpos anti-TPO y anti-Tg $(p<0.001)$.

Es destacable que aproximadamente $40 \%$ de los pacientes con hipotiroidismo fue positivo para
Tabla 1. Características demográficas, pruebas de función tiroidea $y$ anticuerpos antitiroideos en pacientes con artritis reumatoide y controles clínicamente sanos

\begin{tabular}{|c|c|c|c|c|c|}
\hline \multirow[t]{2}{*}{ Característica } & \multicolumn{4}{|c|}{ Media \pm DE } & \multirow[t]{2}{*}{$p$} \\
\hline & \multicolumn{2}{|c|}{$\begin{array}{c}\text { AR } \\
(n=78)\end{array}$} & \multicolumn{2}{|c|}{$\begin{array}{c}\text { CCS } \\
(n=81)\end{array}$} & \\
\hline Edad & \multicolumn{2}{|c|}{$42.75 \pm 12.15$} & \multicolumn{2}{|c|}{$41.80 \pm 11.61$} & 0.75 \\
\hline $\begin{array}{l}\text { Duración de la } \\
\text { enfermedad (años) }\end{array}$ & \multicolumn{2}{|c|}{$4.74 \pm 6.04$} & \multicolumn{2}{|c|}{-} & - \\
\hline Anti-TPO (UI/mL) & \multicolumn{2}{|c|}{$118.26 \pm 538.60$} & \multicolumn{2}{|c|}{$19.18 \pm 55.28$} & 0.02 \\
\hline \multirow[t]{2}{*}{ Anti-Tg (UI/mL) } & \multicolumn{2}{|c|}{$96.94 \pm 321.27$} & \multicolumn{2}{|c|}{$54.99 \pm 46.43$} & 0.04 \\
\hline & $\mathrm{n}$ & $\%$ & $\mathrm{n}$ & $\%$ & \\
\hline Sexo femenino & 65 & 83.3 & 75 & 92.6 & 0.77 \\
\hline Anti-TPO positivos & 12 & 15.4 & 4 & 10.5 & 0.09 \\
\hline Anti-Tg positivos & 10 & 19.6 & 5 & 13.2 & 0.57 \\
\hline \multicolumn{6}{|l|}{ Función tiroidea } \\
\hline Eutiroidismo & 59 & 75.6 & 80 & 98.7 & 一 \\
\hline Hipotiroidismo & 19 & 24.4 & 1 & 1.2 & 0.003 \\
\hline Hipertiroidismo & 0 & & 0 & & - \\
\hline \multicolumn{6}{|l|}{ Ultrasonido tiroideo } \\
\hline Ecogenicidad normal & 52 & 66.6 & 47 & 71.2 & - \\
\hline Nódulos & 18 & 23.0 & 19 & 28.4 & 0.13 \\
\hline Hipoplasia & 2 & 2.8 & 0 & & - \\
\hline Perfusión incrementada & 6 & 7.6 & 1 & 1.5 & - \\
\hline \multicolumn{6}{|l|}{ TIRADS } \\
\hline 1 & 54 & 69.2 & 75 & 92.6 & - \\
\hline 2 & 12 & 15.4 & 3 & 3.7 & - \\
\hline 3 & 5 & 6.4 & 2 & 2.5 & 0.01 \\
\hline $4 a$ & 6 & 7.7 & 1 & 1.2 & - \\
\hline $4 b$ & 1 & 1.3 & 0 & & - \\
\hline
\end{tabular}

Anti-TPO = anti-peroxidasa tiroidea, Anti-Tg = antitiroglobulina, US = ultrasonido; TIRADS = Thyroid Imaging Reporting and Data System. Las comparaciones entre proporciones fueron analizadas con chi cuadrado o prueba exacta de Fisher. Las comparaciones entre los promedios se llevaron a cabo mediante t de Student para muestras no pareadas.

anticuerpos anti-TPO y anti-Tg. En los resultados del US de acuerdo con la función tiroidea: se encontró $70 \%$ con ecogenicidad normal, mientras que $53 \%$ presentó hipotiroidismo $(p<0.001)$ (Tabla 3$)$.

Se realizó biopsia de glándula tiroidea a siete pacientes que clasificaron en TIRADS 4 (4a-4b); las lesiones fueron benignas (datos no mostrados). La duración de la enfermedad fue de 7.14 años (rango de 1 a 22).

\section{Discusión}

Si bien se encontró alta frecuencia de hipotiroidismo en pacientes con AR comparados con CCS ( $24.4 \%$ versus $1.2 \%, p=0.003$ ), en $89.5 \%$ de estos 
Tabla 2. Comparaciones de las características de los pacientes con artritis reumatoide en relación con la presencia o ausencia de anticuerpos antitiroideos

\begin{tabular}{|c|c|c|c|c|c|c|c|c|c|c|}
\hline \multirow[t]{2}{*}{ Característica } & \multicolumn{2}{|c|}{$\begin{array}{c}\text { Anti-TPO (+) } \\
(n=12)\end{array}$} & \multicolumn{2}{|c|}{$\begin{array}{c}\text { Anti-TPO (-) } \\
(n=66)\end{array}$} & p & \multicolumn{2}{|c|}{$\begin{array}{l}\text { Anti-Tg }(+) \\
(\mathrm{n}=10)\end{array}$} & \multicolumn{2}{|c|}{$\begin{array}{l}\text { Anti-Tg }(-) \\
(n=68)\end{array}$} & \multirow[t]{2}{*}{$p$} \\
\hline & \multicolumn{2}{|c|}{ Media \pm DE } & \multicolumn{2}{|c|}{ Media \pm DE } & & \multicolumn{2}{|c|}{ Media \pm DE } & \multicolumn{2}{|c|}{ Media \pm DE } & \\
\hline Duración de la enfermedad (años) & \multicolumn{2}{|c|}{$4.94 \pm 6.64$} & \multicolumn{2}{|c|}{$3.38 \pm 2.66$} & 0.32 & \multicolumn{2}{|c|}{$5.37 \pm 7.17$} & \multicolumn{2}{|c|}{$2.97 \pm 2.73$} & 0.31 \\
\hline \multirow[t]{2}{*}{ DAS28 (unidades) } & \multicolumn{2}{|c|}{$3.12 \pm 1.23$} & \multicolumn{2}{|c|}{$3.14 \pm 1.03$} & 0.97 & \multicolumn{2}{|c|}{$3.02 \pm 1.06$} & \multicolumn{2}{|c|}{$3.51 \pm 1.48$} & 0.36 \\
\hline & $\mathrm{n}$ & $\%$ & $\mathrm{n}$ & $\%$ & & $\mathbf{n}$ & $\%$ & $\mathrm{n}$ & $\%$ & \\
\hline Factor reumatoide positivo & 9 & 75.0 & 24 & 36.4 & 0.03 & 6 & 60.0 & 24 & 35.3 & 0.25 \\
\hline Anti-PCC positivo & 10 & 83.3 & 31 & 47.0 & 0.04 & 8 & 80.0 & 29 & 42.6 & 0.06 \\
\hline \multicolumn{11}{|l|}{ Ultrasonido tiroideo } \\
\hline Ecogenicidad normal & 3 & 25.0 & 49 & 74.2 & $<0.001$ & 4 & 40.0 & 51 & 75.0 & 0.07 \\
\hline Nódulos & 2 & 16.7 & 16 & 24.3 & 0.60 & 0 & & 16 & 23.5 & 0.01 \\
\hline Hipoplasia & 2 & 16.7 & 0 & & 0.01 & 2 & 20.0 & 0 & & 0.01 \\
\hline Perfusión incrementada & 5 & 41.6 & 1 & 1.5 & $<0.001$ & 4 & 40.0 & 1 & 1.5 & $<0.001$ \\
\hline \multicolumn{11}{|l|}{ TIRADS } \\
\hline 1 & 4 & 33.3 & 50 & 75.8 & $<0.001$ & 3 & 10.0 & 51 & 77.9 & 0.07 \\
\hline 2 & 4 & 33.3 & 8 & 12.1 & 0.78 & 3 & 10.0 & 9 & 16.2 & 0.67 \\
\hline 3 & 4 & 33.3 & 1 & 1.5 & 0.001 & 4 & 30.0 & 1 & 2.9 & $<0.001$ \\
\hline $4 a$ & 0 & & 6 & 9.1 & 0.28 & 0 & & 6 & 8.8 & 0.33 \\
\hline $4 b$ & 0 & & 1 & 1.5 & 0.67 & 0 & & 1 & 1.5 & 0.70 \\
\hline
\end{tabular}

DAS28 = índice de actividad de la enfermedad 28, anti-PCC = antipéptido cíclico citrulinado, Anti-TPO = antiperoxidasa tiroidea, Anti-Tg = antitiroglobulina, TIRADS = Thyroid Imaging Reporting and Data System. Las comparaciones entre proporciones se realizaron mediante chi cuadrado o prueba exacta de Fisher. Las Comparaciones de los promedios fueron analizadas con t de Student para muestras no pareadas.

pacientes fue subclínico y franco en $10.5 \%$. De acuerdo con otros reportes, la prevalencia de hipotiroidismo subclínico a nivel mundial es de 3 a $15 \%$ y en AR, de 2.5 a $10.7 \% .11,21,22$

Los niveles de anticuerpos antitiroideos fueron más elevados en pacientes versus CCS (anti-TPO $p=0.02$ y anti-Tg $p=0.04$ ).

En los pacientes con hipotiroidismo, la frecuencia de anti-TPO (36.8 \% versus $8.5 \%, p=0.05)$ y anti-Tg ( $42.1 \%$ versus $3.4 \%, p=0.01)$ fue más alta en comparación con los eutiroideos.

Una de las ETA con mayor prevalencia es la TH, caracterizada por disfunción tiroidea, ecogenicidad difusa incrementada de la glándula tiroides, infiltrado linfocítico en biopsia y anticuerpos antitiroideos, especialmente anti-TPO en $90 \% .{ }^{23-25}$ Buchanan et al. demostraron asociación entre $\mathrm{TH}$ y $\mathrm{AR},{ }^{26}$ sin embargo, en nuestra investigación no se presentó.

Siete mujeres con AR y una CCS fueron clasificadas como TIRADS 4. Todas fueron sometidas a biopsia por aspiración; el resultado fue tiroiditis aguda leve, condición benigna relacionada con ETA. Es importante destacar la posible contribución de las hormonas femeninas en el desarrollo de bocio en la tiroides, nódulos y cáncer en las mujeres. ${ }^{27}$
En relación con los hallazgos de TIRADS 4, es importante recordar las limitaciones del sistema de clasificación TIRADS 4, ya que el riesgo de malignidad en esta categoría específica es muy amplio. ${ }^{17,18}$ Una limitación de nuestro estudio fue su naturaleza transversal. Se necesitarán estudios prospectivos con un gran número de pacientes para determinar si todos los casos de tiroiditis aguda leve en AR se mantienen benignos o progresan a cáncer de tiroides. ${ }^{28}$

El sistema TIRADS se desarrolló para establecer las principales características que predicen malignidad en la enfermedad tiroidea nodular. ${ }^{17}$ En el presente trabajo, los nódulos tiroideos fueron identificados en $23 \%$ del grupo de AR versus $28.4 \%(p=0.13)$ en el grupo de CCS; esta prevalencia concuerda con reportes previos. ${ }^{11,29}$ La biopsia de los nódulos tiroideos indicaron una clasificación TIRADS 4a-4b, lo que traduce un riesgo de malignidad de 5 a $10 \%$ y de 10 a $80 \%$, respectivamente. ${ }^{19}$

EI US tiroideo es un método asequible y sensible que puede utilizarse durante la consulta ambulatoria, además, es capaz de identificar pequeñas lesiones parenquimatosas de 1 a $2 \mathrm{~mm}$, así como cambios relacionados con la autoinmunidad tiroidea, no necesariamente asociados con anticuerpos antitiroideos o disfunción de 
Gaceta Médica de México. 2018;154

Tabla 3. Comparaciones de las características entre pacientes con artritis reumatoide con y sin disfunción tiroidea

\begin{tabular}{|c|c|c|c|c|c|}
\hline \multirow[t]{2}{*}{ Característica } & \multicolumn{4}{|c|}{ Media \pm DE } & \multirow[t]{2}{*}{$\mathrm{p}$} \\
\hline & \multicolumn{2}{|c|}{ Eutiroidismo $(n=59)$} & \multicolumn{2}{|c|}{ Hipotiroidismo $(n=19)$} & \\
\hline Edad (años) & \multicolumn{2}{|c|}{$41.79 \pm 12.54$} & \multicolumn{2}{|c|}{$43.75 \pm 7.10$} & 0.75 \\
\hline Duración de la enfermedad (años) & \multicolumn{2}{|c|}{$4.41 \pm 6.45$} & \multicolumn{2}{|c|}{$5.30 \pm 6.39$} & 0.69 \\
\hline DAS28, unidades & \multicolumn{2}{|c|}{$3.11 \pm 1.26$} & \multicolumn{2}{|c|}{$3.39 \pm 1.04$} & 0.47 \\
\hline Anti-PCC (UI/mL) & \multicolumn{2}{|c|}{$114.76 \pm 224.93$} & \multicolumn{2}{|c|}{$111.14 \pm 72.06$} & 0.93 \\
\hline Anti-TPO (UI/mL) & \multicolumn{2}{|c|}{$31.26 \pm 27.13$} & \multicolumn{2}{|c|}{$310.38 \pm 633.73$} & 0.01 \\
\hline Anti-Tg (UI/mL) & \multicolumn{2}{|c|}{$18.40 \pm 19.17$} & \multicolumn{2}{|c|}{$417.85 \pm 1052.51$} & 0.02 \\
\hline \multirow[t]{2}{*}{ Factor reumatoide $(\mathrm{UI} / \mathrm{mL})$} & \multicolumn{2}{|c|}{$143.83 \pm 313.25$} & \multicolumn{2}{|c|}{$55.62 \pm 67.12$} & 0.12 \\
\hline & $\mathbf{n}$ & $\%$ & $\mathbf{n}$ & $\%$ & \\
\hline Anti-PCC positivo & 27 & 45.8 & 6 & 31.6 & 0.21 \\
\hline Factor reumatoide positivo & 19 & 32.2 & 9 & 47.4 & 0.10 \\
\hline Anti-TPO positivo & 5 & 8.5 & 7 & 36.8 & 0.05 \\
\hline Anti-Tg positivo & 2 & 3.4 & 8 & 42.1 & 0.01 \\
\hline \multicolumn{6}{|l|}{ Ultrasonido tiroideo } \\
\hline Ecogenicidad normal & 42 & 71.2 & 10 & 52.6 & $<0.001$ \\
\hline Nódulos & 10 & 17.0 & 8 & 42.1 & 0.12 \\
\hline Hipoplasia & 1 & 1.7 & 1 & 5.3 & 0.72 \\
\hline Perfusión incrementada & 6 & 10.1 & 0 & & 0.08 \\
\hline \multicolumn{6}{|l|}{ TIRADS } \\
\hline 1 & 41 & 69.5 & 13 & 68.3 & 1.00 \\
\hline 2 & 8 & 13.6 & 4 & 21.1 & 0.43 \\
\hline 3 & 4 & 6.8 & 1 & 5.3 & 0.60 \\
\hline $4 a$ & 5 & 8.5 & 1 & 5.3 & 0.64 \\
\hline $4 b$ & 1 & 1.6 & 0 & & 0.59 \\
\hline
\end{tabular}

DAS28 = índice de actividad de la enfermedad 28, anti-PCC = antipéptido cíclico citrulinado, FR = factor reumatoide, Anti-TPO = antiperoxidasa tiroidea, Anti-Tg = antitiroglobulina, TIRADS = Thyroid Imaging Reporting and Data System. Las comparaciones entre las proporciones fueron realizadas con chi cuadrado o mediante la prueba exacta de Fisher. Las comparaciones entre promedios fueron analizadas con $t$ de Student para muestras no pareadas.

la hormona tiroidea; podría detectar estadios preclínicos de disfunción tiroidea en la AR.

Con base en nuestros hallazgos, los pacientes con AR pueden beneficiarse de la evaluación tiroidea por US, debido a que $10 \%$ de los pacientes clasificó en TIRADS 4 a pesar de ser negativo para anticuerpos antitiroideos y presentar función tiroidea normal.,

Dado el alto número de pacientes con hipotiroidismo subclínico, sugerimos revisión anual de las pruebas de la función tiroidea, exámenes precisos y económicamente accesibles.

\section{Bibliografía}

1. Vázquez-Del Mercado $M$, Nuñez-Atahualpa L, Figueroa-Sánchez $M$ Gómez-Bañuelos E, Rocha-Muñoz AD, Martín-Márquez BT, et al. Serum levels of anticyclic citrullinated peptide antibodies, interleukin-6, tumor necrosis factor-alpha, and C-reactive protein are associated with increased carotid intima-media thickness: a cross-sectional analysis of a cohort of rheumatoid arthritis patients without cardiovascular risk factors. Biomed Res Int. 2015;2015:342649.

2. McInnes IB, Schett $G$. The pathogenesis of rheumatoid arthritis. N Engl J Med. 2011;365(23):2205-2219.

3. Dong YH, Fu DG. Autoimmune thyroid disease: mechanism, genetics and current knowledge. Eur Rev Med Pharmacol Sci. 2014;18(23): 3611-3618.

4. Cárdenas Roldán J, Amaya-Amaya J, Castellanos-De-La-Hoz J, Giraldo-Villamil J, Montoya-Ortiz G, Cruz-Tapias P, et al. Autoimmune thyroid disease in rheumatoid arthritis: a global perspective. Arthritis. 2012;2012:864907.

5. Robazzi TC, Adan LF. Autoimmune thyroid disease in patients with rheumatic diseases. Rev Bras Reumatol. 2012;52(3):417-430.

6. Shiroky JB, Cohen M, Ballachey ML, Neville C. Thyroid dysfunction in rheumatoid arthritis: a controlled prospective survey. Ann Rheum Dis. 1993;52(6):454-456

7. Atzeni F, Doria A, Ghirardello A, Turiel M, Batticciotto A, Carrabba M, et al. Anti-thyroid antibodies and thyroid dysfunction in rheumatoid arthritis: prevalence and clinical value. Autoimmunity. 2008;41(1):111-115.

8. Przygodzka M, Filipowicz-Sosnowska A. Prevalence of thyroid diseases and antithyroid antibodies in women with rheumatoid arthritis. Pol Arch Med Wewn. 2009;119(1-2):39-43.

9. Yavasoglu I, Senturk T, Coskun A, Bolaman Z. Rheumatoid arthritis and anti-thyroid antibodies. Autoimmunity. 2009;42(2):168-169.

10. Andonopoulos AP, Siambi V, Makri M, Christofidou M, Markou C, Vagenakis $A G$. Thyroid function and immune profile in rheumatoid arthritis. A controlled study. Clin Rheumatol. 1996;15(6):599-603. 
11. Mobini M, Kashi Z, Ravanbakhsh N. Thyroid disorders in rheumatoid arthritis and osteoarthritis. Pak J Med Sci. 2011;27(3):595-598.

12. Del Puente A, Savastano S, Nuzzo V, Esposito A, Lupoli G. High prevalence of thyroid autoantibodies in newly diagnosed rheumatoid arthritis patients. Clin Exp Rheumatol. 2003;21(1):137.

13. Arnett FC, Edworthy SM, Bloch DA, McShane DJ, Fries JF, Cooper NS et al. The American Rheumatism Association 1987 revised criteria for the classification of rheumatoid arthritis. Arthritis Rheum. 1988:31(3):315-324.

14. Aletaha D, Neogi T, Silman AJ, Funovits J, Felson DT, Bingham CO, et al. 2010 rheumatoid arthritis classification criteria: an American College of Rheumatology/European League against Rheumatism Collaborative Initiative. Arthritis Rheum Dis. 2010;69(9):2569-2581.

15. Prevoo ML, Van-'t-Hof MA, Kuper HH, Van-Leeuwen MA, Van-De-Putte LB, Van-Riel PL. Modified disease activity scores that include twenty-eight-joint counts. Development and validation in a prospective longitudinal study of patients with rheumatoid arthritis. Arthritis Rheum. 1995;38(1):44-48.

16. Baloch Z, Carayon P, Conte-Devolx B, Demers LM, Feldt-Rasmussen U, Henry JF, et al. Laboratory medicine practice guidelines. Laboratory support for the diagnosis and monitoring of thyroid disease. Thyroid. 2003;13(1):123-126.

17. Horvath E, Majlis S, Rossi R, Franco C, Niedmann JP, Castro A, et al. An ultrasonogram reporting system for thyroid nodules stratifying cancer risk for clinical management. J Clin Endocrinol Metab. 2009;94(5):1748-1751.

18. Kwak JY, Han KH, Yoon JH, Moon HJ, Son EJ, Park SH, et al. Thyroid imaging reporting and data system for US features of nodules: a step in establishing better stratification of cancer risk. Radiology. 2011; 260(3):892-899.
19. Cibas ES, Ali SZ. The Bethesda system for reporting thyroid cytopathology. Thyroid. 2009;19(11):1159-1165.

20. Gharib H, Papini E, Paschke R, Duick DS, Valcavi R, Hegedüs L, et al. American Association of Clinical Endocrinologists, Associazione Medici Endocrinologi, and European Thyroid Association medical guidelines for clinical practice for the diagnosis and management of thyroid nodules: executive summary of recommendations. Endocr Prac. 2010;16(3): 468-475.

21. Fatourechi V. Subclinical hypothyroidism: an update for primary care physicians. Mayo Clin Proc. 2009;84(1):65-71

22. Peeters RP. Subclinical Hypothyroidism. N Engl J Med. 2017;377(14):1404

23. Mincer DL, Jialal I. Thyroid, Hashimoto thyroiditis. Treasure Island (FL): StatPearls Publishing; 2018.

24. Iddah MA, Macharia BN. Autoimmune thyroid disorders. ISRN Endocrinol. 2013;2013:509764.

25. Engler H, Riesen WF, Keller B. Diagnostic value of autoantibodies against microsomal thyroid peroxidase (anti-TPO). Schweiz Med Wochenschr. 1992;122(51-52):1976-1980.

26. Buchanan WW. The relationship of Hashimoto's thyroiditis to rheumatoid arthritis. Geriatrics. 1965;20(11):941-948.

27. Hartmann K. Thyroid disorders in the oncology patient. J Adv Pract Oncol. 2015;6(2):99-106.

28. Li H, Li J. Thyroid disorders in women. Minerva Med. 2015;106(2):109-114.

29. Cooper DS, Doherty GM, Haugen BR, Kloos RT, Lee SL, Mandel SJ, et al. Revised American Thyroid Association management guidelines for patients with thyroid nodules and differentiated thyroid cancer. Thyroid. 2009;19(11):1167-1214. 